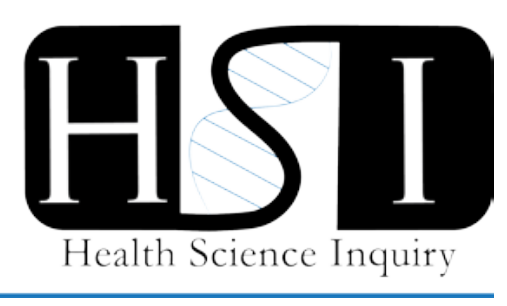

\title{
Catheter re-use: thrifty or threatening? A commentary on intermittent catheter re-use by individuals with spinal cord injury.
}

\author{
Anna Rudkovsa ${ }^{1}$, Yoah Sui ${ }^{2}$, Marisa Kfrerer ${ }^{1}$ \\ ${ }^{1}$ Department of Health \& Rehabilitation Sciences, University of Western Ontario; ${ }^{2}$ Department of Kinesiology, \\ University of Western Ontario
}

Spinal cord injury $(\mathrm{SCl})$ is a temporary or chronic condition resulting from damage to the spinal cord which can be sustained traumatically or due to long lasting spinal degeneration. Changes in muscle tone, sensation, and autonomic function (including bladder dysfunction) are often experienced by those with $\mathrm{SCl}$. Bladder dysfunction is experienced by almost all individual living with $\mathrm{SCl}$ [1] and can often be physically, medically, and socially debilitating. Some forms of bladder dysfunction include: hyperflexic bladder, where the bladder is spastic and may void spontaneously; or flaccid bladder, in which it is difficult to void and may lead to bladder damage from overfilling [2]. For those living with $\mathrm{SCl}$, intermittent catheterization (IC) is often one of the few options available for bladder voiding and offers a safe, clean, and relatively easy way to void the bladder and is often associated with gaining greater independence [3]. In this mini-review, the issue of catheter reuse due to financial constraints will be discussed. Suggestions for future research directions will also be provided.

During IC, a thin, hollow tube is manually inserted into the urethra to help empty the bladder. The procedure can be carried out by the individual themselves, or with the assistance of a nurse. In many developing countries, including Canada, catheters used for IC are not included under the medical coverage umbrella [4]. Outof-pocket costs for IC may be a major contributing factor to catheter re-use by individuals with $\mathrm{SCl}$. Catheter reuse is associated with an elevated risk for urinary tract infections (UTIs) and higher risks for urethral trauma [4], but for many individuals living with $\mathrm{SCl}$, still remains the only viable option for somewhat clean catheterization [4]. According to an article by Woodbery et al. 2008, $50 \%$ of participants surveyed in Canada reported reusing catheters. Although single catheter use and the use of more expensive, pre-lubricated catheters are associated with an almost $20 \%$ decrease in UTI risk (compared to $70-80 \%$ UTI prevalence rate with catheter re-use in observational studies), individuals are still willing to re-use catheters [4]. Single-use catheters, on average, cost $\$ 46 /$ week [6]. Annually, this can add up to a hefty $\$ 2,392$ (cost for purchasing single use, pre-lubricated catheters), which may be out of reach for individuals of low socioeconomic status. A cost for simply using the bathroom.

Catheter re-use is also associated with increased risk for damage to the urethra. Urethral trauma is most common with the use of non-lubricated catheters (which also tend to be cheaper; [6]. Irritation and damage to the urethra may contribute to increased risk of UTIs and other bacterial infections [5]. Although proper cleaning and re-sterilization of a catheter is necessary in preventing bacteria entering the bladder and reducing the risk for UTIs, current cleaning techniques can range from running the catheter under lukewarm water to the use of household bleach, or other antiseptic solutions. Proper cleaning and sterilization technique for the re-use of catheters has yet to be established and it is unknown whether such technique would be feasible and effective in household settings, due to the complexity of procedure or potential costs associated with the necessary cleaning solutions [7]. Participants from the Hakansson article, for example, reported sterilizing catheters anywhere from daily to weekly, with greatly varying methods [4].

Future research should focus on collaboration with policy makers and work towards better medical coverage that would include catheters for individuals with $\mathrm{SCl}$. Research focusing on associated health risks and proper cleaning techniques may be beneficial as leverage when negotiating policy and medical coverage. In addition, future findings may be used to educate policy makers and other stakeholders on the risks of catheter re-use, bringing the issue of catheter re-use and its associated health risks to the forefront. In the mean time, patient education must remain an important component of the rehabilitation process. Education about the risks of catheter re-use should be clearly conveyed to patients by their nurses and other health care practitioners. Although, unfortunately, many will still re-use catheters due to financial constraints. 
Living with an $\mathrm{SCl}$ can already prove to be challenging experience; however, it should not be a limiting factor to the quality of life, independence, and health an individual may attain. Simply requiring IC should not be directly associated with avoidable health risks. More work needs to be done at education, research, and policy levels to assure the highest possible standard of health for those living with $\mathrm{SCl}$.

\section{References}

[1] SCIRE Community Team. Spinal Cord Injury Basics [Internet]. SCIRE Community. 2017. Available from: https://scireproject.com/community/topic/sci-basics/

[2] 2. Hsieh, J., Mclntyre, A., Iruthayarajah, J., Loh, E., Ethans, K., Mehta, S., Wolfe, D., Teasell, R. (2014). Bladder Management Following Spinal Cord Injury. In SCIREV 5. 0., p 1-196. Bladder Management. Available from: https://scireproject.com/evidence/rehabilitationevidence/bladder-management/
[3] Newman, D. K., Willson, M. M. (2011) BT-UN. Review of intermittent catheterization and current best practices. Urologic Nursing, 31 (1), 12.

[4] Hakansson, M. A. (2014) Reuse versus single-use catheters for intermittent catheterization: What is safe and preferred? Review of current status. Spinal Cord, 52 (7), 511516.

[5] Woodbury, M. G., Hayes, K. C., Askes, H. K. (2008) Intermittent catheterization practices following spinal cord injury: a national survey. Can J Urol., 15 (3), 40654071.

[6] Prieto, J., Murphy, C. L., Moore, K. N., Fader, M. (2014) Intermittent catheterisation for longterm bladder management (abridged cochrane review). Neurourol Urodyn, 34 (7), 648653. Available from: https://doi.org/10.1002/nau.22792

[7] Sherbondy, A. L., Cooper, C. S., Kalinowski, S. E., Boyt, M. A., Hawtrey, C. E. (2002) Variability in Catheter Microwave Sterilization Techniques in a Single Clinic Population. J Urol, 168 (2), 562564. 

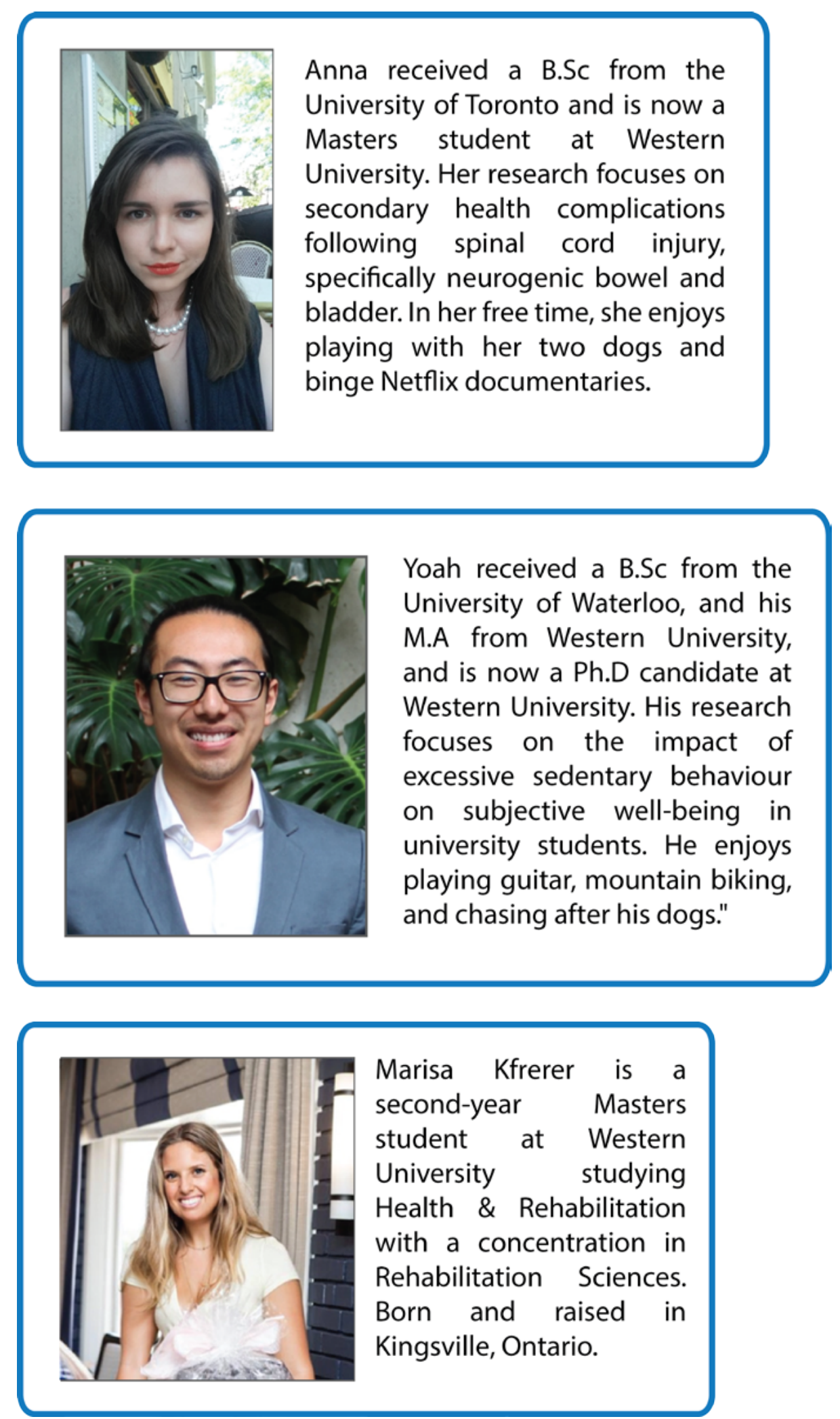